\title{
What's new since Hippocrates? Preventing type 2 diabetes by physical exercise and diet
}

\author{
J. A. Hawley • M. J. Gibala
}

Received: 23 August 2011 /Accepted: 9 December 2011 / Published online: 21 January 2012

(C) Springer-Verlag 2012

\begin{abstract}
Since the work of Eriksson and Lindgärde, published over two decades ago (Diabetologia 1991;34:891898), we have known that type 2 diabetes can be prevented or delayed by supervised lifestyle interventions (physical exercise and diet modification) in persons at risk of the disease. Here we discuss a novel, time-efficient approach to physical exercise prescription, low-volume, high-intensity interval training (LVHIT), and its efficacy for inducing a range of health benefits in a variety of populations at risk of inactivity-related diseases. We look to the future and suggest that current guidelines for exercise may need to be revised to include different training techniques to deliver the optimum exercise prescription. Indeed, we predict that subsequent exercise guidelines will include LVHIT as part of a comprehensive 'fitness menu' that allows individuals to select the exercise regimen that best fulfils their medical needs, is suited to their lifestyle and daily time restraints, and meets their personal goals.
\end{abstract}

Keywords Continuous glucose monitoring · Exercise guidelines · Exercise prescription - Low-volume, high-intensity interval training

\section{Abbreviations \\ IGT Impaired glucose tolerance \\ HVHIT High-volume, high-intensity interval training}

\section{J. A. Hawley $(\bowtie)$}

Exercise Metabolism Group,

Health Innovations Research Institute,

School of Medical Sciences, RMIT University,

PO Box 71, Bundoora, VIC 3083, Australia

e-mail: john.hawley@rmit.edu.au

M. J. Gibala

Department of Kinesiology, McMaster University,

Hamilton, ON, Canada L8S 4K1
LVHIT Low-volume, high-intensity interval training

$\dot{V} \mathrm{O}_{2 \max } \quad$ Maximal oxygen uptake

\section{Background}

The recent proliferation in the rate of diagnosis of a constellation of metabolic disorders (including obesity, insulin resistance and type 2 diabetes) stems from the willingness of most individuals in industrialised nations to adopt a sedentary lifestyle in the face of an abundant food supply and excess energy intake. While five decades of evidence from epidemiological, experimental and clinical trials support a positive relationship between dietary intake and disease risk, only recently has it been demonstrated that a physically inactive lifestyle initiates a cascade of cellular events that are mechanistically linked to a plethora of chronic metabolic disease states [1]. The view that physical inactivity and excess energy intake are directly linked to the rising incidence of metabolic diseases is, however, hardly new, and the notion that these conditions can be prevented or postponed by lifestyle interventions is by no means a modern-day concept. The Greek physician, Hippocrates (460 BC to $370 \mathrm{BC}$ ) was one of the first to postulate that disease is the product of environmental factors, namely diet and living habits (and not, as widely believed at the time, a punishment inflicted by 'the gods or evil spirits'). Unlike most other physicians of his day, Hippocrates believed that diseases had a physical origin and that good health depended on proper diet, plenty of exercise, fresh air and good personal hygiene. He was the first to categorise illnesses as being acute, chronic, endemic and epidemic. Unfortunately, medicinal therapy at the time of Hippocrates was an immature science and often the only thing a 
physician could do for a patient was evaluate their condition and predict its likely progression (often death) based on data collected from detailed case histories.

Medical science has evolved somewhat since the time of the ancient Greeks, and promotion of physical activity coupled with a healthy diet is now commonplace in national and international guidelines as primary therapy for preventing type 2 diabetes. But just how much progress have we made since the writings of Hippocrates in successfully implementing interventions that prevent or delay chronic metabolic diseases? And what are the best ways to promote permanent behavioural changes in the level of habitual physical activity and diet in individuals at risk of developing type 2 diabetes?

\section{Then: the work of Eriksson and Lindgärde and others}

Over 20 years ago Diabetologia published the results of a 'feasibility study' that had its origins in two age-cohort screening programmes undertaken in the city of Malmö, Sweden, between 1974 and 1985 [2]. The primary aim of that investigation [2] was to determine if the beneficial effects of short-term (6-12 months) lifestyle interventions could be extended over a longer time-frame (5-6 years) in approximately 200 individuals with impaired glucose tolerance (IGT) or type 2 diabetes. In short, the answer was 'yes'. Indeed, probably one of the most important outcomes from the study by Eriksson and Lindgärde [2] was that such a large group of sedentary, overweight, middle-aged, glucose-intolerant or diabetic individuals successfully completed a 5 year intervention with a dropout rate of less than $10 \%$. Such compliance was, in part, achieved by regular counselling, supervised physical training and frequent medical check-ups. Details pertaining to specifics of the exercise and/or dietary modifications were, however, not addressed in the report, making it hard to ascertain just how much exercise participants undertook, or the extent of their dietary modifications [2]. Notwithstanding this lack of information, the effect of the exercise-diet interventions on several physiological indices were impressive: maximal oxygen uptake $\left(\dot{V} \mathrm{O}_{2 \max }\right.$, the index used as a marker of fitness) was increased by $10-14 \%$ in participants with IGT after lifestyle intervention compared with a 5-9\% reduction in the control (non-intervention) group $(p<0.001)$. Body mass was reduced by $2.3-2.7 \%$ among active participants, whereas values increased by $0.5-1.7 \%$ in control participants who were glucose tolerant, as well as in individuals with IGT who did not participate in any intervention $(p<0.05)$. Weight reduction and improved fitness were correlated with improved glucose tolerance, with subjects who both lost weight and increased fitness improving the most. Both blood lipid profile and blood pressure were reduced, and early insulin responsiveness to glucose loading was preserved in the lifestyle intervention group. The bottom line was that more than $50 \%$ of the diabetic patients were in remission after a mean follow-up of 6 years [2].

Since the work of Eriksson and Lindgärde [2], at least three large-scale, multi-centre, randomised clinical trials of lifestyle interventions have been published [3-5]. These studies have involved between 500 and 3,000 overweight men and women with IGT, and the prescription of mild- to moderate-intensity weight-bearing aerobic exercise, typically undertaken several times a week, for prolonged periods (3-5 years). In one study [5], resistance training was also incorporated into the lifestyle intervention programme. In the other studies, exercise regimens were combined with and/or compared with diet [4] or drug intervention [3]. The results of these landmark studies have previously been reviewed in detail [6], and taken collectively, strongly support the hypothesis that type 2 diabetes can be prevented or delayed by lifestyle interventions in persons at risk of the disease.

\section{Now: current public health guidelines for the prevention and treatment of metabolic diseases}

Interventions to promote changes in diet and/or physical activity in adults with an increased risk of type 2 diabetes are more likely to be effective if they target both these lifestyle factors, involve the planned use of established behavioural change techniques and have a clear plan for supporting maintenance of behaviour change [7]. While this sounds simple in theory, nothing could be further from the truth. With regard to current guidelines for physical activity, leading public health agencies, including the ADA, typically recommend at least $150 \mathrm{~min}$ per week of moderate to vigorous aerobic-based exercise to prevent and treat chronic metabolic diseases [8]. As we have previously discussed, such guidelines are based on incontrovertible evidence from observational and randomised clinical trials demonstrating that regular physical activity contributes to the primary and secondary prevention of type 2 diabetes, cardiovascular disease and all-cause mortality [2-5]. Indeed, there is a robust dose-response relationship between the amount of exercise performed (from $~ 2.9$ $8.4 \mathrm{~kJ}$ of energy expenditure per week) and all-cause mortality and cardiovascular disease mortality in middle-aged and elderly populations [9]. However, despite overwhelming proof that an inactive lifestyle (coupled with excess energy intake) leads to a host of chronic diseases and premature death, the majority of adults fail to meet minimum physical activity guidelines. The most commonly cited barrier to performing regular exercise, regardless of sex, age, ethnicity or health status is a lack of time [10]. In addition, adherence to exercise programmes comprising continuous bouts of prolonged exercise is poor unless there is an adequate level of supervision 
and appropriate infrastructure [2]. Taken collectively, these issues highlight the need for innovative, time-efficient, clinically effective exercise strategies, coupled with changes in current attitudes and methods of delivering exercise prescription in order to improve metabolic health and reduce the risk of type 2 diabetes.

\section{Looking ahead: time for large-scale, multi-centre, randomised clinical trials of high-intensity exercise training?}

Part of the reason for the growing apathy to modify lifestyle habits is that current public health recommendations may be unrealistic and unattainable for the majority of the populace. In our opinion, it seems highly improbable that individuals who presently fail to undertake $30 \mathrm{~min}$ of continuous exercise will ever choose to perform double the amount of the same activity, which would be the amount required to substantially reduce their all-cause mortality and cardiovascular disease risk [9]. Here we 'look ahead' and suggest that the current guidelines for exercise prescription may need to be revised to include different training techniques to deliver the optimum exercise prescription. We propose an alternative, time-efficient approach to exercise prescription in an effort to encourage greater compliance. This strategy, which has received recent scientific attention [11-13], is generically referred to as high-intensity interval training and is characterised by brief bursts of intense effort interspersed by short periods (intervals) of recovery. The efficacy of highvolume, high-intensity interval training (HVHIT) protocols (requiring a time commitment similar to traditional endurance training) for improving disease outcomes has been demonstrated in several studies of clinical populations, including overweight adolescents, obese adults, and patients with the metabolic syndrome, heart failure and coronary artery disease (reviewed in [13]). Although results from these investigations demonstrate the effectiveness of

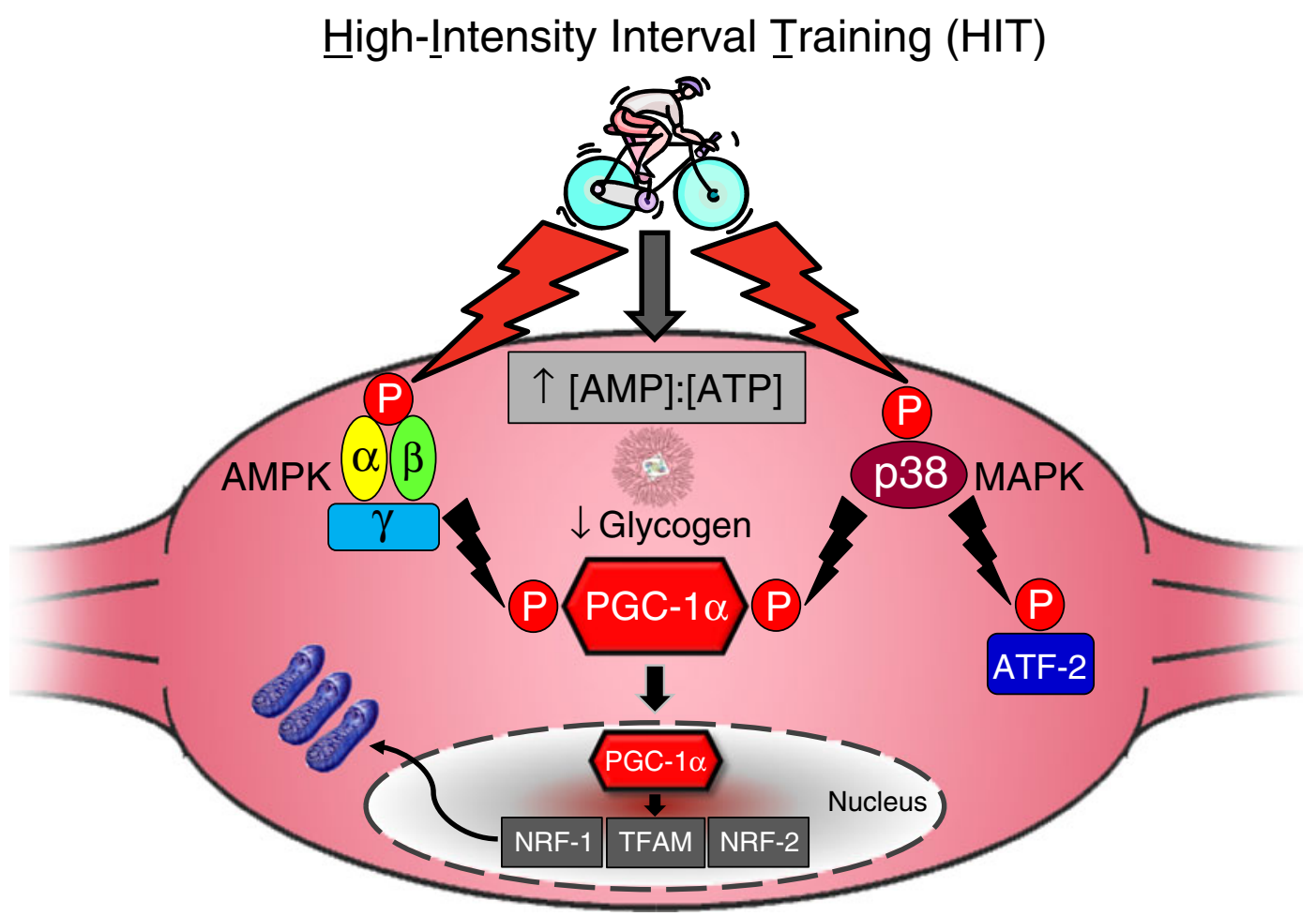

Fig. 1 Putative signalling cascades promoting skeletal muscle mitochondrial biogenesis in response to high-intensity interval training (HIT). HIT causes a greater increase in the ratio of the concentration of AMP to ATP, and also a faster rate of muscle glycogenolysis compared with continuous, submaximal endurance exercise. These intracellular perturbations result in the rapid activation (through phosphorylation $[\mathrm{P}]$ ) of the AMP-activated protein kinase (AMPK) and the p38 mitogen-activated protein kinase (MAPK). Both AMPK and the p38 MAPK phosphorylate and activate the peroxisome proliferatoractivated receptor- $\gamma$ coactivator (PGC-1 $\alpha)$. The p38 MAPK also increases PGC- $1 \alpha$ expression by phosphorylating activating transcription factor 2 (ATF-2), which increases $\mathrm{PGC}-1$ protein production by binding to and activating the CREB site on the $P G C-1 \alpha$ promoter. PGC- $1 \alpha$ in the nucleus docks on, and activates, several transcription factors, including nuclear-respiratory factor 1 (NRF-1) and nuclearrespiratory factor 2 (NRF-2), which bind to the promoters and activate transcription of the genes that encode mitochondrial respiratory chain proteins. NRF-1 also activates expression of the nuclear gene that encodes mitochondrial transcription factor A (TFAM), which moves to the mitochondria, where it regulates transcription of the mitochondrial DNA (i.e. the mitochondrial genome). It is likely that the initial phase of the increase in mitochondrial biogenesis induced by HIT is mediated by activation of PGC- $1 \alpha$, while the second phase is mediated by an increase in $\mathrm{PGC}-1 \alpha$ protein production 
HVHIT for individuals with chronic diseases, such exercise protocols still require a considerable time investment by the participant. In contrast, a growing body of evidence suggests that low-volume, high-intensity interval training (LVHIT) is just as potent a stimulus for inducing healthpromoting adaptations as traditional high-volume endurance training, despite a dramatically reduced time commitment. For example, several weeks of LVHIT in both young healthy individuals [14] and middle-aged adults [15] results in similar increases in skeletal muscle mitochondrial capacity as endurance training, despite a $75 \%$ reduced time commitment and a $90 \%$ lower training volume. These are important findings because skeletal muscle mitochondrial content is reduced in inactive individuals with insulin resistance, and may play a permissive role in the development of type 2 diabetes [16]. A summary of the putative signalling cascades involved in skeletal muscle mitochondrial biogenesis in response to high-intensity interval training is shown in Fig. 1.

Several studies that have utilised LVHIT have had the disadvantage that the extremely demanding protocols, often consisting of 'all out' cycling (e.g. repeated $30 \mathrm{~s}$ Wingate Tests), may not be safe or tolerable for clinical populations. Accordingly, we have recently tested an alternative protocol consisting of $10 \times 60 \mathrm{~s}$ intense (but not maximal) constantload cycle bouts (eliciting about $90 \%$ of maximal heart rate $\left.\left[\mathrm{HR}_{\max }\right]\right)$, interspersed with $60 \mathrm{~s}$ of recovery. This protocol is time efficient: only $10 \mathrm{~min}$ of exercise is performed in a $20 \mathrm{~min}$ training session. And it is highly effective for inducing rapid changes in both skeletal muscle and whole body indices of health: six training sessions over a 2 week period improved insulin sensitivity (assessed using fasting insulin and glucose measurements [insulin sensitivity index scores determined using the HOMA method]) in previously sedentary middle-aged adults who may have been at high risk of developing insulin resistance and type 2 diabetes [15]. We have also recently reported that an LVHIT protocol can rapidly improve skeletal muscle oxidative capacity, GLUT4 content and glycaemic control in patients with type 2 diabetes [17]. In this study, continuous glucose monitoring systems were used to provide real-time data about the magnitude and frequency of daily blood glucose excursions under free-living conditions [17] (Fig. 2). Such information revealed that the mean $24 \mathrm{~h}$ blood glucose concentration was reduced after only 2 weeks of training (from $\sim 7.6-$ $6.6 \mathrm{mmol} / 1, p<0.05$ ), as were the sum of the $3 \mathrm{~h}$ postprandial areas under the glucose curve after breakfast, lunch and dinner $(p<0.05)$. These improvements in health outcomes were evident despite the total training time being some $60 \%$ lower than recently revised guidelines calling for $150 \mathrm{~min}$ per week to promote health [8].

The results from a recent study [18] also suggest that high-intensity interval training confers greater benefits on the heart compared with distance-matched, moderate-

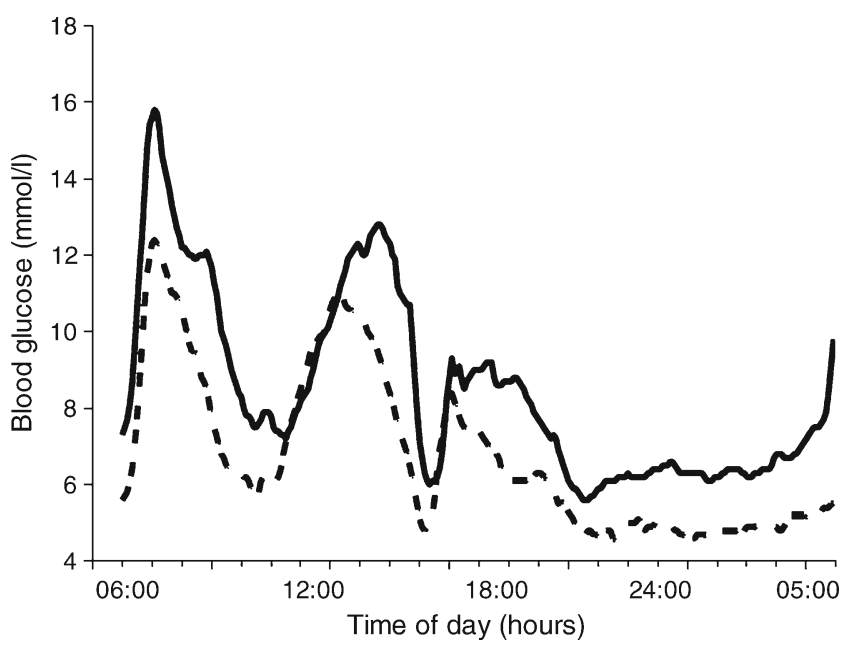

Fig. 2 Blood glucose concentration determined from continuous glucose monitoring (CGM) in a representative patient over the course of a day ( $24 \mathrm{~h}$ ) before (pre; solid line) and after (post; dashed line) 2 weeks of high-intensity interval training (HIT, see text for further details). Post-training CGM data were collected $\sim 48-72 \mathrm{~h}$ after the final exercise training session. Figure reproduced from [17] with permission

intensity continuous treadmill running. Mice performing HVHIT (4 min at $85-90 \%$ of $\dot{V} \mathrm{O}_{2 \max }$ ) had superior metabolic improvements in several markers of myocardial function, including increased cardiac citrate synthase activity, enhanced maximal rates of mitochondrial oxygen consumption in cardiac muscle and greater elevations in the expression of cardiac antioxidant enzymes [18]. The findings of Hafstad and colleagues [18] provide some mechanistic insight as to why intense interval training results in superior cardiovascular adaptations in heart failure patients [19] and why such exercise has tremendous therapeutic potential for patients who were once prescribed bed rest!

\section{Summary and directions for further research}

We are currently losing the battle against chronic metabolic diseases: the incidence of a trio of inactivity-related conditions (obesity, insulin resistance and type 2 diabetes) continues to rise unabated. Despite an impressive body of evidence demonstrating the efficacy of lifestyle interventions (dietary changes and increased physical activity) along with much publicised recommendations from national and international public health authorities, the majority of individuals fail to translate these messages into meaningful actions. The results from recent human studies demonstrate that LVHIT is a potent, time-efficient therapeutic intervention that is more effective than continuous, moderateintensity exercise for (1) improving maximal oxygen uptake in individuals with the metabolic syndrome and patients with heart failure and/or coronary artery disease; (2) lowering fasting and $24 \mathrm{~h}$ blood glucose concentrations in young 
women and patients with type 2 diabetes; (3) reducing body fat in young women; and (4) improving endothelial function in patients with heart failure. Certainly there is no evidence that LVHIT is less effective than traditional endurance exercise guidelines for improving any outcome variable that has currently been measured. Accordingly, we feel the time has come to embark on large-scale, multi-centre, randomised clinical trials to test the effectiveness of LVHIT in a number of clinical populations and on long-term clinical outcome measures. Head-to-head comparisons between current recommendations for exercise prescription (i.e. ADA guidelines) and LVHIT are warranted. Certainly the prescription of such training represents a novel and practical strategy that can be easily incorporated into daily living and reduce the economic burden associated with an inactive lifestyle at both the individual and population level.

Based on the pioneering work of Eriksson and Lindgärde [2] and others [3-5], it is clear that the primary goals of the appropriate medical and legislative bodies should be the promotion of long-term organisation and support of supervised exercise and diet programmes, along with increased education and public awareness of the benefits of a healthy lifestyle. However, in light of the growing body of scientific evidence demonstrating the efficacy of LVHIT for inducing a range of health benefits, we predict that future exercise guidelines will include this type of training as part of a comprehensive "fitness menu' that allows individuals to select the exercise regimen that best fulfils their medical needs, is suited to their lifestyle and daily time restraints, and meets their personal goals. To put our point of view into an historical perspective, in their original position statement on the recommended quantity and quality of exercise for developing and maintaining fitness in healthy adults, published over three decades ago [20], the American College of Sports Medicine recommended a dose of 20-60 min of vigorous exercise, three to five times a week was needed to improve cardiorespiratory fitness. Clearly, we can learn from the past!

Duality of interest The authors declare that there is no duality of interest associated with this manuscript.

Contribution statement JAH and MG were both responsible for the conception of the manuscript, drafting the article and approving the final version for publication.

\section{References}

1. Olsen RH, Krogh-Madsen R, Thomsen C, Booth FW, Pedersen BK (2008) Metabolic responses to reduced daily steps in healthy nonexercising men. JAMA 299:1261-1263
2. Eriksson KF, Lindgärde F (1991) Prevention of type 2 (non-insulindependent) diabetes mellitus by diet and physical exercise. The 6year Malmö feasibility study. Diabetologia 34:891-898

3. Diabetes Prevention Program Research Group (2002) Reduction in the incidence of type 2 diabetes with lifestyle intervention or metformin. N Engl J Med 346:393-403

4. Pan XR, Li GW, Hu YH et al (1997) Effects of diet and exercise in preventing NIDDM in people with impaired glucose tolerance. The Da Qing IGT and diabetes study. Diabetes Care 20:537-544

5. Tuomilehto J, Lindstrom J, Eriksson JG et al (2001) Prevention of type 2 diabetes mellitus by changes in lifestyle among subjects with impaired glucose tolerance. N Engl J Med 344:1343-1350

6. Hawley JA (2004) Exercise as a therapeutic intervention for the prevention and treatment of insulin resistance. Diabetes Metab Res Rev 20:383-393

7. Greaves CJ, Sheppard KE, Abraham C et al (2011) Systematic review of reviews of intervention components associated with increased effectiveness in dietary and physical activity interventions. BMC Publ Health 11:e119

8. Chasan-Taber L, Albright AL, Braun B (2010) Exercise and type 2 diabetes: the American College of Sports Medicine and the American Diabetes Association: Joint position statement. Diabetes Care 33:e147-e167

9. Blair SN, Kohl HW 3rd, Barlow CE, Paffenbarger RS Jr, Gibbons LW, Macera CA (1995) Changes in physical fitness and all-cause mortality: a prospective study of healthy and unhealthy men. JAMA 273:1093-1098

10. Godin G, Desharnais R, Valois P, Lepage L, Jobin J, Bradet R (1994) Differences in perceived barriers to exercise between high and low intenders: observations among different populations. Am J Health Promotion 8:279-284

11. Gibala MJ, Little JP (2010) Just HIT it! A time-efficient exercise strategy to improve muscle insulin sensitivity. J Physiol 588:33413342

12. Hawley JA (2008) Specificity of training adaptation: time for a rethink? J Physiol 586:1-2

13. Gibala MJ (2007) High-intensity interval training: a time-efficient strategy for health promotion? Curr Sports Med Rep 6:211-213

14. Burgomaster KA, Howarth KR, Phillips SM et al (2008) Similar metabolic adaptations during exercise after low volume sprint interval and traditional endurance training in humans. J Physiol 586:151-160

15. Hood MS, Little JP, Tarnopolsky MA, Myslik F, Gibala MJ (2011) Low-volume interval training improves muscle oxidative capacity in sedentary adults. Med Sci Sports Exerc 43:1849-1856

16. Hawley JA, Lessard SJ (2007) Mitochondrial function: use it or lose it. Diabetologia 50:699-702

17. Little JP, Gillen JB, Percival ME et al (2011) Low-volume highintensity interval training reduces hyperglycemia and increases muscle mitochondrial capacity in patients with type 2 diabetes. J Appl Physiol 111:1554-1160

18. Hafstad AD, Boardman NT, Lund J et al (2011) High intensity interval training alters substrate utilization and reduces oxygen consumption in the heart. J Appl Physiol 111:1235-1241

19. Wisloff U, Stoylen A, Loennechen JP et al (2007) Superior cardiovascular effect of aerobic interval training versus moderate continuous training in heart failure patients: a randomized study. Circulation 111:3086-3094

20. American College of Sports Medicine (1978) Position statement on the recommended quantity and quality of exercise for developing and maintaining fitness in healthy adults. Med Sci Sports Exerc 10:vii-x 